\title{
Fluorescence and Electroluminescence of J-Aggregated Polythiophene Monolayers on Hexagonal Boron Nitride
}

\author{
James Kerfoot,* Simon A. Svatek, Vladimir V. Korolkov, Takashi Taniguchi, Kenji Watanabe, \\ Elisa Antolin, and Peter H. Beton*
}

Cite This: ACS Nano 2020, 14, 13886-13893

Read Online

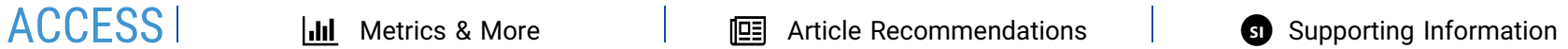

ABSTRACT: The photophysics of a semiconducting polymer is manipulated through molecular self-assembly on an insulating surface. Adsorption of polythiophene (PT) monolayers on hexagonal boron nitride (hBN) leads to a structurally induced planarization and a rebalancing of inter- and intrachain excitonic coupling. This conformational control results in a dominant 0-0 photoluminescence peak and a reduced Huang-Rhys factor, characteristic of $\mathrm{J}$ type aggregates, and optical properties which are significantly different to both PT thin films and single polymer strands. Adsorption on $\mathrm{hBN}$ also provides a route to explore electroluminescence from PT monolayers though incorporation into hybrid van der Waals heterostructures whereby the polymer monolayer is embedded within a hBN tunnel diode. In these structures we observe up-converted singlet electroluminescence from the PT monolayer, with an excitation mechanism based upon inelastic electron scattering. We argue that surface adsorption provides a methodology for the study of fundamental optoelectronic properties of technologically relevant polymers.

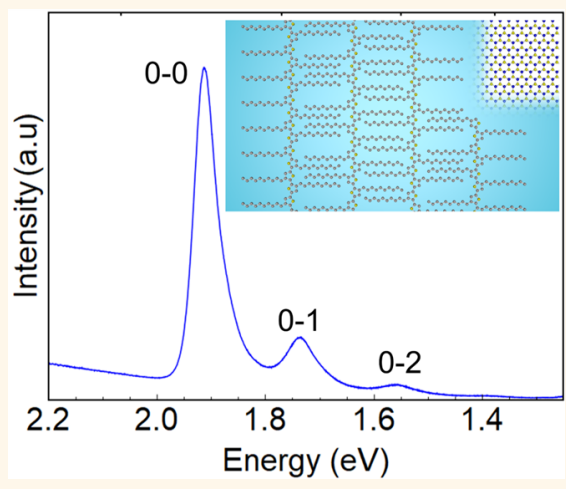

KEYWORDS: self-assembly, photoluminescence, electroluminescence, semiconducting polymer, organic electronics, molecular electronics, photophysics

r he environment and interchain interactions of polymeric semiconductors are important determinants in the electronic and photophysical properties of their associated devices and thin films. ${ }^{1-3}$ Semiconducting polymers offer significant advantages over their small-molecule counterparts $^{4,5}$ due to increased conjugation which enhances intrachain exciton and charge transport. ${ }^{6,7}$ However, the mechanical flexibility inherent to polymer chains leads to a range of structural conformations which can both limit intrachain coupling and cause variations in the optical properties of single, isolated chains. ${ }^{8-10}$ An investigation of the fundamental properties of polymer chains would ideally require a configuration in which both the conformation and the interaction between neighboring chains can be independently controlled, but in practice, this is very difficult to achieve. For example, in thin films of polythiophene (PT), one of the most extensively studied polymers, the polymer backbone is planarized in a lamellar structure, but the resulting optical properties are strongly influenced by interactions with neighboring molecules. ${ }^{11,12}$ Consequently, the effects of planarization, which can control the degree of conjugation, and H-type aggregation, arising from cofacial polymer packing, are hard to disentangle. ${ }^{12-14}$ Effects due to nearest neighbors can be eliminated in studies of single PT molecules in a frozen matrix, ${ }^{15-17}$ but in this configuration polymers are in uncontrolled, nonplanar conformations in the absence of side-chain engineering approaches which can produce deterministic backbone configurations. ${ }^{18,19}$ It is therefore highly desirable to identify a configuration in which the polymer conformation can be controlled, but decoupled from interchain interactions, thus allowing investigations of fundamental optoelectronic polymer properties. We show below that the adsorption of polymer monolayers on a planar insulating surface provides a route to the realization of this goal.

Received: July 27, 2020

Accepted: September 8, 2020

Published: September 8, 2020 
The optical properties of organic polymers can be greatly affected by $\mathrm{H}$ - and J-type aggregation, determined by the relative interplay of inter- and intrachain interactions. ${ }^{13}$ Polymerization leads to conjugated arrays of fluorophores, where the excitonic coupling within the polymer chain arises due to both through-bond and electrostatic dipole-dipole interactions. ${ }^{6,7,10}$ Intrachain coupling between subunits leads to J-type aggregation, ${ }^{6}$ in which coherent coupling between chromophores shifts the exciton band to lower energy for the optically allowed transition at $k=0$, where $k$ is the exciton wave vector. This leads to a red shift in the emission maximum and an enhancement in photoluminescence intensity, ${ }^{20,21}$ benefiting applications requiring bright, and spectrally sharp, luminescence. In contrast to intrachain interactions, interchain interactions within aggregates of organic polymers often lead to H-type aggregation, where coupling between neighboring molecules shifts the exciton energy at $k=0$ to higher energy, leading to an indirect bandstructure and suppression of the direct $0-0$ optical transition. ${ }^{13,22}$

It has recently been shown that $\mathrm{H}$ - and J-type aggregation, and therefore the optical properties, of small organic molecules can be controlled through the variation of their relative position and orientation within self-assembled domains which form on the surface of hexagonal boron nitride ( $\mathrm{hBN}$ ) and are stabilized, for example, through hydrogen-bonding interactions. $^{23-26}$ Molecular monolayers adsorbed on supporting flakes of hBN, an insulating 2D material, can also be integrated into hybrid van der Waals heterostructures, forming tunnel diodes, which enable the electroluminescence from the molecular layers to be explored, ${ }^{25,27}$ resulting in various effects such as photon up-conversion and selective spin triplet excitation. ${ }^{28-32}$ Polythiophene can also be adsorbed on the surface of hBN; the polymer backbone can be resolved within monolayer-thick islands using atomic force microscopy under ambient conditions allowing the molecular scale characterization of these self-assembled monolayers. ${ }^{33}$ In light of these results, it is interesting to consider whether the order imparted by adsorption influences the optical properties of a polymer as previously reported for small molecules. 4,34

In this paper, we show that the self-assembly of polymeric monolayers on an insulating surface provides a route to an environment which is complementary to both thin films and single molecules frozen in a matrix and, moreover, allows characterization of conformation at the molecular scale. Specifically, excitonic coupling, and hence the luminescence, of semiconducting polymers can be explored without the need for $\pi$-stacking of molecules ${ }^{22}$ or side chain engineering. ${ }^{17,18}$ By depositing self-assembled monolayers of the PT derivative poly[3-decylthiophene-2,5-diyl] (P3DT) on hBN it is possible to form aggregates exhibiting the photophysical properties of Jtype aggregates, which reveal a much lower Huang-Rhys factor and more extended vibronic structure than has previously been reported for polythiophene. ${ }^{15-17,35}$ It is also possible to integrate these polymeric monolayers into van der Waals tunnel diodes, allowing the electron-induced generation of excitons and electroluminescence from an embedded monolayer of polythiophene. We believe that this approach could be extended to a wide range of technologically relevant semiconducting polymers and provide further insight into the photophysical properties of these important materials.

\section{RESULTS}

Photoluminescence from Monolayer P3DT Aggregates on hBN. After deposition from solution onto precleaned hBN substrates (see the Methods), P3DT forms monolayer islands with a lamellar structure, as depicted in Figure la,b, due to the interdigitation of the decyl side groups

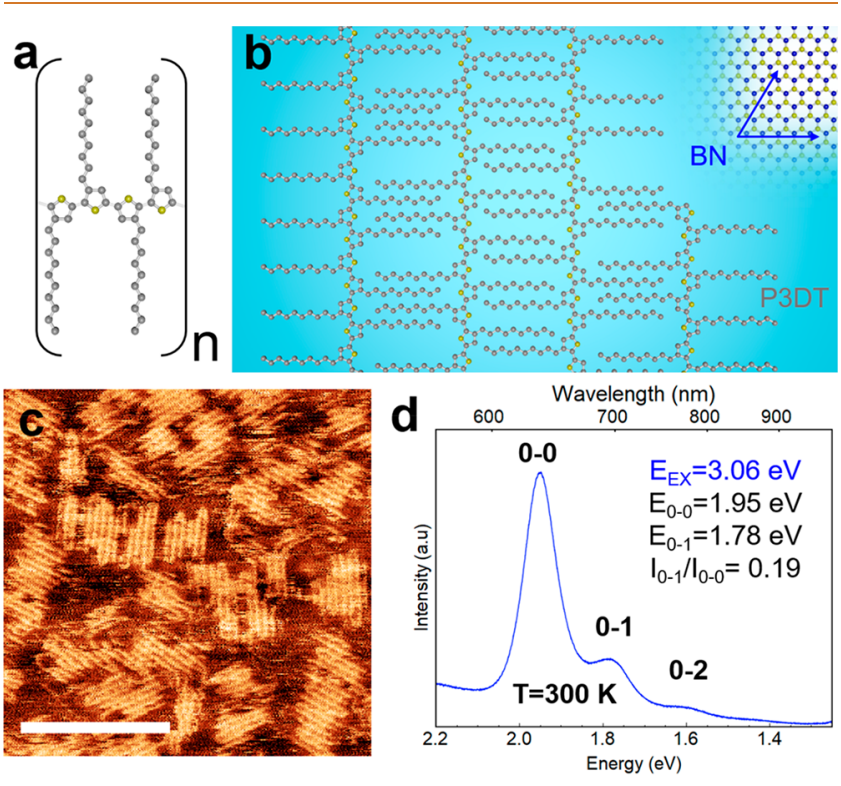

Figure 1. Photoluminescence of monolayer polythiophene on hBN. Schematic diagrams of the molecular structure of the deposited polythiophene (poly[3-decylthiophene-2,5-diyl]) (a) and its packing arrangement on hBN (the lattice and lattice vectors are overlaid on the top right-hand corner), which is driven by the interdigitation of alkane chains of neighboring polythiophene backbones and their alignment with the principal axes of hBN (b). Images of the morphology (scale bar $40 \mathrm{~nm}$ ) of a polythiophene film on $\mathrm{hBN}$, deposited by immersion (see the Methods) were acquired using AFM (c) and reveal the presence of monolayer-thick polythiophene assemblies with a fractional surface coverage of 0.68 . The photoluminescence spectrum of the film was acquired at room temperature using a $405 \mathrm{~nm}$ excitation source (c), showing a strong zero-phonon peak and clearly resolved vibronic sidebands. Inset: peak positions.

attached to the polythiophene backbone. ${ }^{33}$ As described in our previous work, atomic force microscopy (AFM) can be used under ambient conditions to resolve individual polymer chains and determine the morphology of self-assembled P3DT. ${ }^{33}$ The AFM images in Figure 1c show P3DT islands of monolayer thickness with the polymer backbone parallel to the substrate. From the AFM images we determine a fractional surface coverage of 0.68 , with no evidence of multilayers. We also observe a number of "kinks" in polymer chains at the edges of islands, whereby polymer chains are bent through $180^{\circ}$. The extent of straight polythiophene sections, those between either the end of a chain or a "kink", was determined to be $12.0 \pm 4.6$ $\mathrm{nm}$. An average number of 7-8 such parallel sections were found per polythiophene island, separated by a distance of 1.99 $\pm 0.05 \mathrm{~nm}$.

The room-temperature photoluminescence spectra of a monolayer P3DT film (see Methods; excitation source photon energy $E_{\mathrm{EX}}=3.06 \mathrm{eV}$ ) show an intense $0-0$ peak at $E_{0-0}=$ $1.95 \mathrm{eV}$, with clearly resolved $0-1$ and $0-2$ vibronic satellite peaks at, respectively, $E_{0-1}=1.78 \mathrm{eV}$ and $E_{0-2}=1.59 \mathrm{eV}$, see 
Figure $1 \mathrm{~d}$. The intensities, $I_{0-0}$ and $I_{0-1}$, of, respectively, the $0-$ 0 and $0-1$ peaks, were extracted by fitting to a FranckCondon progression and reveal a ratio, $I_{0-1} / I_{0-0}=0.19$, significantly lower than thicker polythiophene films $\left(I_{0-1} / I_{0-0}\right.$ $>1),{ }^{12}$ single chains in a frozen matrix $\left(I_{0-1} / I_{0-0} \sim 0.7\right)^{17}$ and J-aggregated polythiophene nanofibers $\left(I_{0-1} / I_{0-0} \sim 0.5\right) .{ }^{36}$ The measured ratio is also significantly lower than the value, $\sim 1$, of poly(3-hexylthiophene) (P3HT) in solution. ${ }^{11}$ In addition, we measure a $0-2 / 0-1$ peak ratio, $I_{0-2} / I_{0-1}=0.15$ at $300 \mathrm{~K}$, with a minimum of $I_{0-2} / I_{0-1}=0.10$ at $120 \mathrm{~K}$, which is significantly lower than that reported for P3HT thin films, ${ }^{12} I_{0-2} / I_{0-1} \sim 0.5$ (the ratio $I_{0-2} / I_{0-1}$ has been related to an effective HuangRhys factor, $S_{\text {eff }}=2 I_{0-2} / I_{0-1}$, by Paquin et al. ${ }^{12}$ who argued that this parameter is more sensitive to intrachain coupling than $\left.I_{0-1} / I_{0-0}\right)$. For P3DT aggregates on $\mathrm{hBN}$, the low values observed for the $I_{0-1} / I_{0-0}$ peak ratio and effective HuangRhys factor are attributed to the adsorption geometry of P3DT in which polymers lie flat on the substrate in islands of monolayer thickness with no overlayers. This configuration is expected to lead to planarization of the polymer backbone and thus enhanced intrachain J-type aggregation, ${ }^{6,15,37}$ while suppressing the effects of interchain coupling which lead to H-type aggregation.

In order to explore the influence of conformation and morphology on the resonant interactions determining $\mathrm{H}$ - and J-type aggregation, the temperature dependence of the P3DT photoluminescence was measured as shown in Figure 2. At temperatures down to $6 \mathrm{~K}$, the $0-0$ peak remains dominant and the vibronic satellite peaks can be resolved up to the $0-3$ peak. The $0-0$ and associated vibronic peaks were red-shifted by, respectively, 43 and $53 \mathrm{meV}$ between 300 and $6 \mathrm{~K}$, with a reduction in the full width half-maximum of the $0-0$ peak from 86 to $38 \mathrm{meV}$, see Figure 2, but minimal change in peak intensity, see the Supporting Information. The approximate linear dependence of red-shift on temperature (see Figure 2b) suggests increasing exciton diffusion to lower energy sites at low temperature. ${ }^{1,38}$ The gradient of the red-shift of the $0-0$ peak, $0.13 \mathrm{meV} / \mathrm{K}$, is smaller than the value for isolated chains of polythiophene derivatives $(0.2-0.5 \mathrm{meV} / \mathrm{K})$, indicating that the local planarity of the polymer adsorbed on $\mathrm{hBN}$ is greater than that of single strands in a frozen matrix..$^{10,17,39}$ At low temperature, vibronic peaks in the photoluminescence spectra up to the 0-3 index were resolved; the energy separation of successive vibronic peaks, $\Delta E=0.177 \pm 0.001 \mathrm{eV}$ at $6 \mathrm{~K}$ extracted by fitting to a Franck-Condon progression (fitted peaks are shown in SI), is close to the energy associated with the vinyl stretch of the polythiophene backbone. ${ }^{40}$

The measured ratio of the intensities of the $0-0$ peak and its 0-1 vibronic satellite peak (see Figure 2c) reveals a weak temperature dependence which contradicts the expected enhancement of the $0-0$ peak relative to the $0-1$ peak for Jtype aggregates, ${ }^{6,22,41}$ suggesting a more complicated balance between inter- and intrachain interactions. The strength of interchain and intrachain coupling, respectively, $J_{\text {inter }}$ and $J_{\text {intra }}$ can be estimated from the temperature dependence of the $I_{0-0} / I_{0-1}$ peak ratio using the $\mathrm{H} / \mathrm{J}$-type aggregate model. ${ }^{13}$ The peak ratio has a maximum value at $\left(I_{0-0} / I_{0-1}\right)_{\max }$ at a temperature $T_{\max }$ and according to this model, $4 F J_{\text {inter }}=$ $K_{\mathrm{B}} T_{\max }$, where $F$ is the Franck-Condon factor and $\left(I_{0-0} / I_{0-1}\right)_{\max }=1.15 / S_{0} \sqrt{I J_{\text {intra }} \mid / J_{\text {inter }}}$. The parameter $S_{0}$ is the Huang-Rhys factor which characterizes the strength of vibrational coupling for thiophene units in the noninteracting
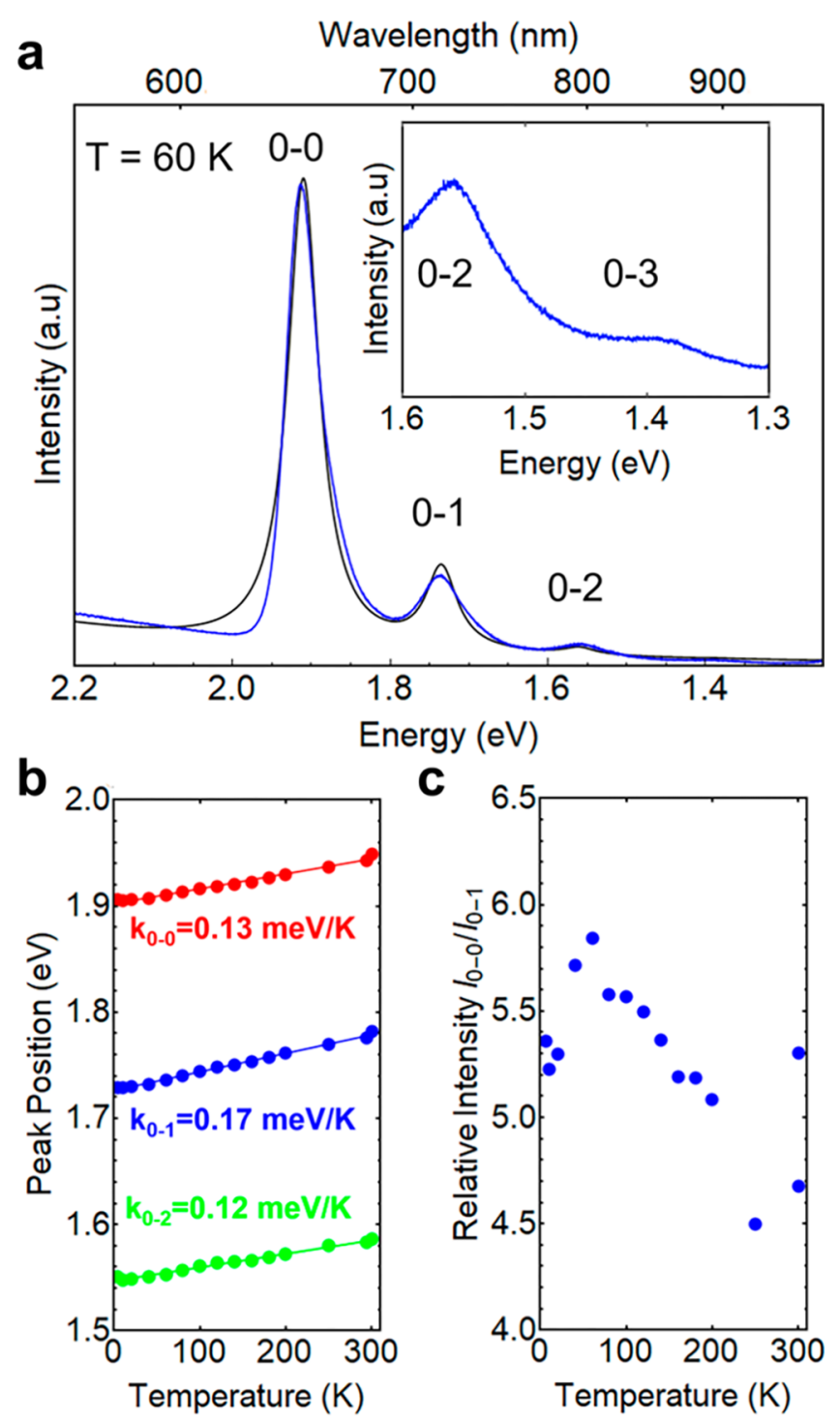

Figure 2. Temperature dependence of photoluminescence of polythiophene on $\mathrm{hBN}$. Measurements acquired at $60 \mathrm{~K}$ show a narrowing of the $0-0$ peak with respect to room temperature measurements (a). Vibronic satellite peaks up to the 0-3 peak, inset, could be resolved. The positions, separations, and relative intensities of these peaks were extracted from fitting to a FranckCondon progression of Lorentzian curves, plotted in black. The position and relative intensities of the $0-0,0-1$, and $0-2$ peaks over the whole temperature range from 6 to $300 \mathrm{~K}$ are shown plotted in panels (b) and (c), respectively.

limit (i.e., assuming that changes to the fluorescence line shape due to excitonic coupling are neglected). The Huang-Rhys factor of P3HT has been estimated in the literature to be in the range $S_{0}=1-2$ through a comparison of models for intrachain excitonic coupling with data for single chains. ${ }^{12,13,15,36,42}$ Taking the Huang-Rhys factor measured by Clark et al. for a dilute P3HT solution ${ }^{11}$ as a lower bound for $S_{0}$, where $S_{0}=1$ $(F=0.37)$, we obtain $J_{\text {inter }}=3 \pm 1 \mathrm{meV}$ and $J_{\text {intra }}=88 \pm 29$ $\mathrm{meV}$ from experimental values (Figure $2 \mathrm{c}$ ) for $\left(I_{0-0} / I_{0-1}\right)_{\max }=$ 5.8 and $T_{\max }=60 \mathrm{~K}$ for P3DT on hBN.

Our experimental values show an increase in intrachain and decrease in interchain interactions in comparison with $\mathrm{P} 3 \mathrm{HT}$ nanofibers ${ }^{36}$ for which $J_{\text {inter }} \sim 9 \mathrm{meV}$ and $J_{\text {intra }} \sim 60 \mathrm{meV}$, while the interchain coupling is much less than the value $J_{\text {inter }} \sim 30$ 
meV reported for P3HT thin films. ${ }^{11,12}$ In addition, our experimental value for $J_{\text {inter }}$ is close to the estimate, $J_{\text {inter }}=+1$ $\mathrm{meV}$, calculated using the point dipole approximation (we assume a single point dipole interacts with a chain of neighboring dipoles with a chain separation (Figure 1) of 2 $\mathrm{nm}$, a value ${ }^{43}$ for the transition dipole moment of $4 \mathrm{D}$, and taking into account screening from the hBN substrate). ${ }^{24}$

We also estimate the coherence number, $N_{\mathcal{c}}$ from the relationship $I_{0-0} / I_{0-1}=N_{\mathrm{c}} / S_{0}$, ${ }^{6,12,22}$ where we again take $S_{0}=1$ as a lower bound, giving $N_{\mathrm{c}} \sim 5$ and a coherence length, $L_{\mathrm{c}} \sim 4$ $\left(L_{\mathrm{c}}=N_{\mathrm{c}}-1\right)$, of thiophene units over which the exciton is delocalized, equivalent to $\sim 1.6 \mathrm{~nm}$. This value is much less than the typical lengths, $12.0 \pm 4.6 \mathrm{~nm}$, of straight polymer sections (see Figure 1c) confirming that finite size effects are not expected to be significant.

The temperature dependence of the photoluminescence, discussed above, shows that the self-assembly of P3DT on $\mathrm{hBN}$ leads to a conformation which enhances intrachain coupling and suppresses interchain coupling and is consistent with our initial hypothesis that the sharp spectral features are due to planarization of the polymer backbone and the absence of face-to-face packing for the monolayer films we study. Our results show that adsorption of monolayers provides an environment which is complementary to thin films and single molecule studies.

Electroluminescence from P3DT Monolayers in van der Waals Heterostructures. It is also possible to incorporate P3DT monolayers on $\mathrm{hBN}$, similar to those discussed above, into van der Waals heterostructures to produce a hybrid polymer $/ 2 \mathrm{D}$ electrical device. We have adapted a device architecture, which was developed recently to form analogue structures with embedded small organic molecules, to form a heterostructure in which a P3DT monolayer is embedded between two few-layer hBN tunnel barriers and overlapping few-layer graphene (FLG) contacts (see Figure $3 \mathrm{a}) .^{27}$ This device structure is further encapsulated between two thicker ( $>10 \mathrm{~nm}$ ) hBN flakes. Completed devices, as illustrated in Figure $3 \mathrm{~b}$, are constructed in such a way that, under an applied bias, current flows vertically between the fewlayer graphene (FLG) contacts by tunnelling across the $\mathrm{hBN}$ tunnel barriers, between which a P3DT partial (0.68 fractional coverage) monolayer is embedded. This type of device is fabricated by the sequential pick-up and mechanical transfer of flakes of graphene and hBN, including flakes on which the P3DT has been predeposited. The technique is described in Svatek et al. ${ }^{27}$ with more details in SI.

The current-voltage dependence of a completed device consisting of hBN tunnel barriers with thicknesses of $1 \mathrm{ML}$ (top) and $2 \mathrm{ML}$ (bottom), respectively, was acquired at room temperature and is highly nonlinear, as expected for a tunnel diode. $^{27,44-46}$ As the bias, $V$, applied to the device was increased, electroluminescence was observed. The electroluminescence (EL) and photoluminescence (PL) spectra of the encapsulated P3DT show dominant peaks at $E_{\mathrm{EL}}=1.93 \mathrm{eV}$ and $E_{\mathrm{PL}}=1.95 \mathrm{eV}$, respectively, which are broadened with respect to uncapped P3DT monolayers, with the full width half-maximum increasing from 68 to $140 \mathrm{meV}$ between uncapped and capped room temperature photoluminescence measurements; this increase is likely due to variations in the local environment of the encapsulated P3DT within the heterostructures, for example, due to structural defects such as wrinkles and blisters which are common in van der Waals heterostructures. ${ }^{47}$ As shown in Figure $3 \mathrm{~d}$, the vibronic peaks

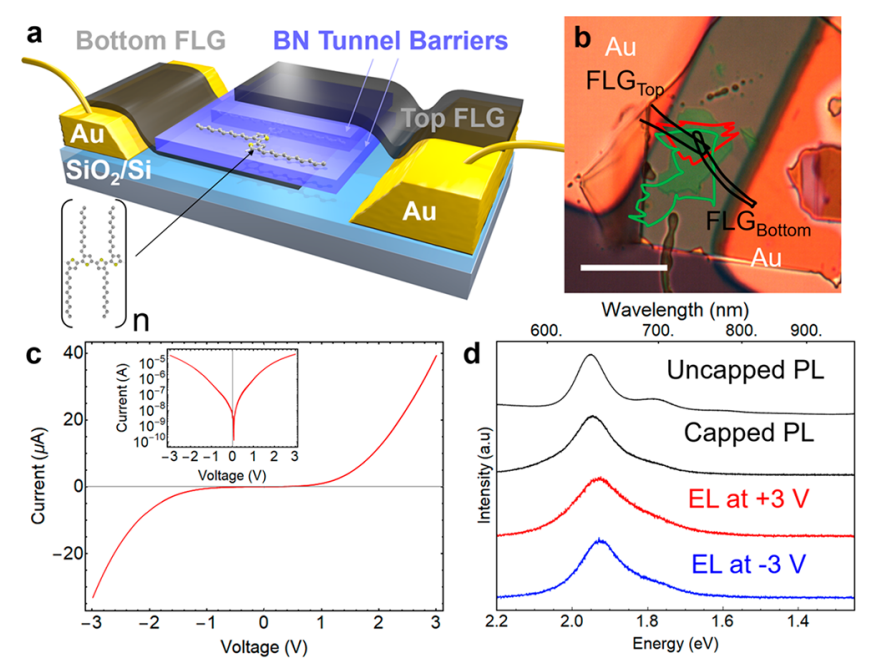

Figure 3. Polymer/hBN hybrid tunnel structure. Using modified transfer techniques for 2D materials, a device architecture consisting of a well-defined monolayer of P3DT embedded between two ultrathin $(<1 \mathrm{~nm})$ hBN tunnel barriers between over- and underlying few layer graphene contacts was fabricated, schematic (a), optical micrograph (b), showing relative position of layers. The current-voltage characteristics of this device were measured (c) and found to be highly nonlinear. Room temperature photoluminescence of capped and uncapped polythiophene and electroluminescence from the completed device were measured (d).

in the photoluminescence and electroluminescence of capped P3DT are less clearly defined than uncapped P3DT. In order to compare with our earlier analysis of vibronic structure, the $I_{0-1} / I_{0-0}$ peak ratio was extracted from the P3DT photoluminescence, $\left(I_{0-1} / I_{0-0}\right)_{\text {Capped-PL }}$ and electroluminescence spectra, $\left(I_{0-0} / I_{0-1}\right)_{\text {Capped-EL }}$ of capped P3DT. We find lower values for these quantities, $\left(I_{0-1} / I_{0-0}\right)_{\text {Capped-PL }}=0.05$ and $\left(I_{0-1} / I_{0-0}\right)_{\text {Capped-EL }}=0.12$, than for uncapped P3DT at room temperature, $\left(I_{0-1} / I_{0-0}\right)_{\mathrm{PL}}=0.19$. It is possible that effects such as pressure and interactions with two hBN interfaces within the heterostructures could lead to changes in the vibrational coupling of $\mathrm{P} \mathrm{DT}{ }^{48}$ however the presence of inhomogeneities, as discussed above, complicate a comparison with homogeneous uncapped P3DT monolayers.

The observation of EL with spectral features which are similar to the PL of capped and uncapped P3DT indicates that charge transport between the two FLG electrodes generates excitons within the P3DT monolayer, which relax though the emission of photons resulting in the measured EL. The evolution of EL with increasing bias measured at $300 \mathrm{~K}$ (in Figure 4) shows no apparent change in the position of the EL peak with increasing voltage. From the anticipated band alignment of the highest occupied molecular orbital $\left(E_{\mathrm{HOMO}}=\right.$ $5 \mathrm{eV})$, the lowest unoccupied molecular orbital $\left(E_{\mathrm{LUMO}}=3\right.$ $\mathrm{eV})$ of P3DT, and the work function of FLG $\left(W_{\mathrm{FLG}}=4.5 \mathrm{eV}\right)$, we rule out direct injection and recombination of electrons and holes as a route to the formation of neutral excitons within the P3DT monolayer; this mechanism would require $V_{\mathrm{EL}} \sim 21$ $W_{\mathrm{FLG}}-E_{\mathrm{LUMO}} / \mathrm{e} \sim 3 \mathrm{~V}$, much larger than the measured onset voltage of EL, $V_{\mathrm{EL}}=1.6 \mathrm{~V}$.

In fact, we observe EL centered at $E_{\mathrm{EL}}=1.93 \mathrm{eV}$ even at the onset voltage, $V_{\mathrm{EL}}=1.6 \mathrm{~V}$; i.e., the photon energy is greater than the energy gained by a single electron traversing the device, $E_{\mathrm{EL}}>\mathrm{e} V_{\mathrm{EL}}$, a process known as photon up-conversion. 


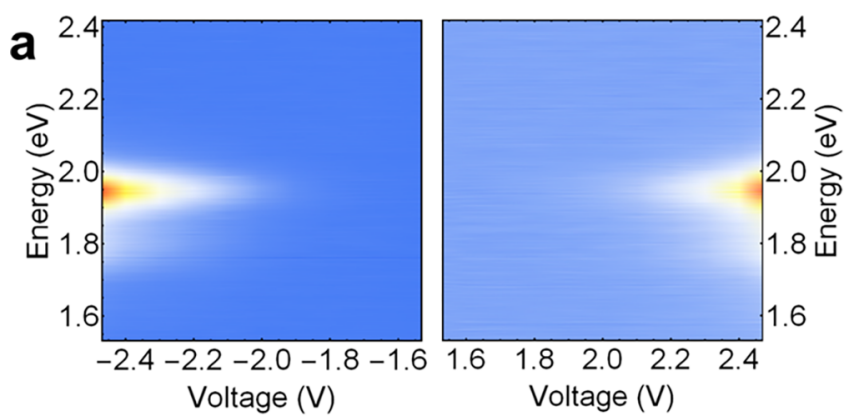

b

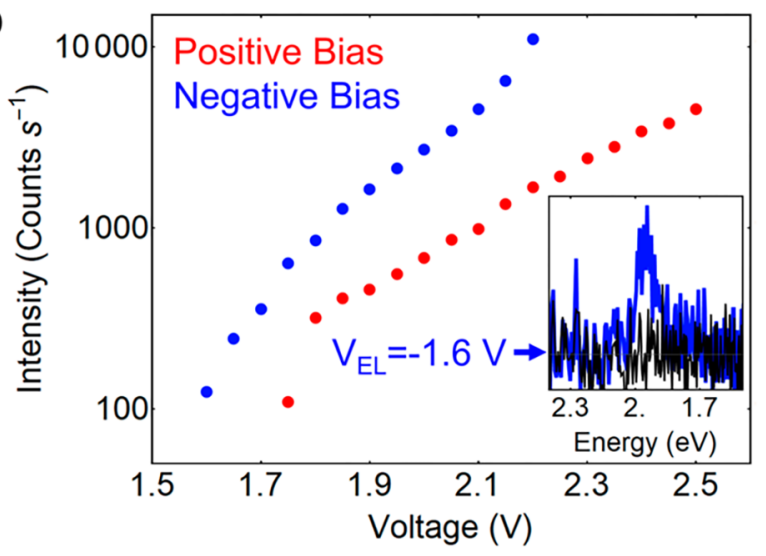

Figure 4. EL of a polythiophene device was measured at $300 \mathrm{~K}$ for a series of applied voltages from 1.5 to $2.5 \mathrm{~V}$ in both forward and reverse bias. The EL intensity is shown plotted against applied voltage and photon energy with separate color scales (a). For a second device, also at $300 \mathrm{~K}$, the total number of counts per second was extracted by integrating background subtracted spectra over the whole wavelength range. A logarithmic plot of the integrated counts versus voltage shows clear subthreshold emission, with the spectrum acquired at $-1.6 \mathrm{~V}$ shown inset for reference.

As discussed in recent papers, ${ }^{27,30,32}$ this indicates that molecules are excited into an intermediate state through inelastic scattering of a tunnelling electron, and then, through a further excitation, into the excited singlet state $S_{1}$. It has been argued that the lifetime of the intermediate state must be longer than the average traversal time, $\tau_{\mathrm{T}}$ of electrons through each fluorophore. We estimate $\tau_{\mathrm{T}} \sim 0.13 \mu \mathrm{s}$ from the measured current density $\left(0.37 \mathrm{pA} \mathrm{nm}^{-2}\right)$ close to the threshold for EL, assuming an effective fluorophore area of $4 \mathrm{~nm}^{2}$. In common with recent studies of EL from organic molecules using scanning tunnelling luminescence, ${ }^{27,30,32}$ and molecular/2D hybrid tunnel devices, ${ }^{27}$ we identify the intermediate state as the spin triplet, $T_{1}$, since other excited states, for example vibrationally excited states, are expected to have much shorter lifetimes. In this scenario, a molecule is excited into a triplet state, $T_{1}$, via inelastic scattering of a tunnelling electron followed by a further excitation to an excited singlet, $S_{1}$, state which relaxes through the emission of a photon. Note that for our observed onset voltage, we measure a photon upconversion, $\left|E_{\mathrm{EL}}-e V_{\mathrm{EL}}\right|$, of approximately $0.35 \mathrm{eV}$, corresponding to $\sim 14 k_{\mathrm{B}} T$ at room temperature, ruling out simple explanations based on thermally excited electrons.

The triplet state of polythiophene is reported to have an energy of approximately $\sim 1.2 \mathrm{eV}^{49}$ Interestingly, we do not observe EL from P3DT in this spectral range, although direct emission from the triplet state has been reported in related studies of some, but not all, small organic mole- cules. $^{27,29,30,32,50}$ The absence of triplet electroluminescence could be due to reduced intersystem crossing rates or more rapid triplet-triplet annihilation (TTA) for P3DT (relative to small molecules such as perylene diimide for which triplet emission is observed) resulting in an EL signal too low to detect using our experimental setup. The mechanism for the $T_{1}$ $\rightarrow S_{1}$ transition may involve a second inelastic tunnelling process, ${ }^{27,32}$ possibly via an initial transition to a higher lying state in the triplet manifold for which it has been shown that intersystem transfer may be more rapid. ${ }^{51}$ Alternatively, this transition may involve a TTA process. ${ }^{52}$ In our recent studies of triplet emission from a small molecule it was possible to rule out TTA as a mechanism, but in the absence of direct triplet emission in our devices we are unable to discriminate between these possible mechanisms and in addition note that Thomas et $a .^{53}$ have shown that the triplet population can be affected by J-type aggregation in $\mathrm{P} 3 \mathrm{HT}$ further complicating a comparison with previous studies of electroluminescence from a molecular monolayer.

The efficiency of our devices $\sim 2 \times 10^{-7}$ photons/electron at $300 \mathrm{~K}$ is comparable to that observed in similar devices and STML experiments. ${ }^{27,30,32,50}$ This low value is at least partially due to the fractional coverage of the P3DT layer (see Figure 1c) of $0.6-0.7$; this coverage was chosen to reduce the occurrence of multilayers and aggregates, while the effective coverage of emissive sites is further reduced by inefficiencies in molecular packing and the area taken up by interdigitated decyl side chains, which do not contribute significantly to the frontier molecular orbitals or light-matter interactions of the polythiophene backbones.

\section{CONCLUSION}

We have shown that the sharp spectral features which we observe for self-assembled P3DT are directly due to the conformation of a polymer monolayer which result from adsorption on hBN which leads to a stabilization of a planar backbone and the absence of face-to-face packing. The adsorbed configuration provides an environment which is complementary to both thin films and single molecules in a frozen matrix. Specifically, adsorption on hBN facilitates the study of the intrinsic optical properties of planarized molecules, eliminating the complications of polythiophene in both thin films, in which planarization occurs due to the lamellar structure but is accompanied by strong interchain coupling leading to $\mathrm{H}$-type aggregation, and as single molecules for which $\mathrm{H}$-type aggregation is eliminated, but the molecules adopt an uncontrolled nonplanar conformation. By incorporating P3DT monolayers into hybrid van der Waals tunnel diodes, we also demonstrate electroluminescence from monolayer thick films of P3DT, where excitations are generated by inelastic scattering of electrons tunnelling across the hybrid $\mathrm{hBN} / \mathrm{P} 3 \mathrm{DT} / \mathrm{hBN}$ junction. The associated observation of photon up-conversion in these devices is both highly relevant to the study of low voltage optoelectronics and provides a route to excite spin-triplet states in conducting polymers. We anticipate that this approach can be applied to a broad range of semiconducting polymers and copolymers offering the possibility to determine fundamental polymeric properties and optimize the bright and fast optical response from atomically thin organic layers. 


\section{METHODS}

Sample Preparation. As in our earlier work, ${ }^{24,33} \mathrm{hBN}$ substrates were prepared by mechanical exfoliation of crystallites onto thermally oxidized $90 \mathrm{~nm} \mathrm{SiO}{ }_{2}$ using the scotch tape method, leaving $\mathrm{hBN}$ flakes on the $\mathrm{SiO}_{2}$ surface with typical lateral sizes and thicknesses of the order of $10 \mu \mathrm{m}$ and $10 \mathrm{~nm}$, respectively. $\mathrm{hBN}$ substrates were cleaned by annealing with a butane gas torch and cooled immediately prior to the deposition of P3DT chains from solution. Regiorandom P3DT (molecular weight 30000-100000) was purchased from American Dye Source (ADS 510) and was dissolved in toluene ( $\geq 99.9 \%$, Sigma-Aldrich) to produce a solution of $2.5 \mu \mathrm{g} \mathrm{mL}^{-1}$ concentration. P3DT films on hBN were prepared by immersion in solution for $45 \mathrm{~s}$ and removal of excess solvent using a nitrogen gun.

Devices were fabricated using a modified version of transfer techniques discussed in the literature and described in our recent work. ${ }^{27,54}$ Using a micromanipulation stage, polypropylene carbonate stamps were used to sequentially pick up a thick hBN flake, FLG, ultrathin hBN and a second ultrathin hBN layer onto which a P3DT had been predeposited. Devices were completed by the transfer of the $\mathrm{hBN} / \mathrm{FLG} / \mathrm{hBN} / \mathrm{P} 3 \mathrm{DT} / \mathrm{hBN}$ stack onto a predeposited heterostructures of $\mathrm{hBN}$ and FLG (stamped from PDMS ${ }^{55}$ ) on predeposited gold contacts on $300 \mathrm{~nm} \mathrm{SiO}$.

Sample Characterization. AFM measurements of the polythiophene film morphology and van der Waals heterostructures at various stages during fabrication were carried out using both the Asylum Research Cypher S and MFP-3D instruments with NuNano Scout 70 probes.

Fluorescence spectroscopy and electroluminescence measurements were carried out using a Horiba MicOS optical spectrometer with a 50X objective (NA: 0.5 ) and a $405 \mathrm{~nm}$ delta diode excitation source with a pulse rate of $100 \mathrm{MHz}$, an average power of approximately 10 $\mu \mathrm{W}$, and a spot size of approximately $2 \mu \mathrm{m}$. For all fluorescence measurements, samples were placed in an Oxford Instruments Microstat $\mathrm{He}$ helium flow cryostat and held under a vacuum of approximately $10^{-6}$ mbar.

Electrical measurements of completed P3DT devices were acquired using a 1612B Keithley Sourcemeter.

\section{ASSOCIATED CONTENT}

\section{(s) Supporting Information}

The Supporting Information is available free of charge at https://pubs.acs.org/doi/10.1021/acsnano.0c06280.

Photoluminescence spectra of P3DT monolayers on hBN from 6 to $300 \mathrm{~K}$, details of the fitting procedures, and an extended description of the device fabrication method (PDF)

\section{AUTHOR INFORMATION}

\section{Corresponding Authors}

James Kerfoot - School of Physics and Astronomy, University of Nottingham, Nottingham NG7 2RD, U.K.; 1 orcid.org/00000002-6041-4833; Email: james.kerfoot@nottingham.ac.uk

Peter H. Beton - School of Physics and Astronomy, University of Nottingham, Nottingham NG7 2RD, U.K.; (i) orcid.org/ 0000-0002-2120-8033; Email: peter.beton@ nottingham.ac.uk

\section{Authors}

Simon A. Svatek - School of Physics and Astronomy, University of Nottingham, Nottingham NG7 2RD, U.K.; Instituto de Energia Solar, Universidad Politécnica de Madrid, Madrid 28040, Spain; 이이이.org/0000-0002-8104-1888

Vladimir V. Korolkov - School of Physics and Astronomy, University of Nottingham, Nottingham NG7 2RD, U.K.

Takashi Taniguchi - International Center for Materials Nanoarchitectonics, National Institute for Materials Science,
Tsukuba 305-0044, Japan; 이이이.org/0000-0002-14673105

Kenji Watanabe - Research Center for Functional Materials, National Institute for Materials Science, Tsukuba 305-0044, Japan; @ orcid.org/0000-0003-3701-8119

Elisa Antolin - Instituto de Energía Solar, Universidad Politécnica de Madrid, Madrid 28040, Spain; (1) orcid.org/ 0000-0002-5220-2849

Complete contact information is available at: https://pubs.acs.org/10.1021/acsnano.0c06280

\section{Notes}

The authors declare no competing financial interest.

The raw data for photoluminescence and electroluminescence spectra, AFM images and electrical measurements may be accessed through the University of Nottingham Research Data Management Repository at http://doi.org/10.17639/nott. 7069 .

\section{ACKNOWLEDGMENTS}

This work was supported by the Engineering and Physical Sciences Research Council (Grant No. EP/N033906/1) and the Leverhulme Trust (Grant No. RPG-2016-104). K.W. and T.T. acknowledge support from the Elemental Strategy Initiative conducted by the MEXT, Japan, Grant No. JPMXP0112101001, JSPS KAKENHI Grant Nos. JP20H00354 and the CREST(JPMJCR15F3), JST. E.A. and S.A.S are grateful for the Ramón y Cajal and Juan de la Cierva Fellowships funded by the Spanish MINECO (RYC-201518539, FJC2018-036517-I). P.H.B. thanks the Leverhulme Trust for the award of a Research Fellowship (RF-2019-460).

\section{REFERENCES}

(1) Eder, T.; Stangl, T.; Gmelch, M.; Remmerssen, K.; Laux, D.; Höger, S.; Lupton, J. M.; Vogelsang, J. Switching between H- and JType Electronic Coupling in Single Conjugated Polymer Aggregates. Nat. Commun. 2017, 8, 1641.

(2) Vilan, A.; Aswal, D.; Cahen, D. Large-Area, Ensemble Molecular Electronics: Motivation and Challenges. Chem. Rev. 2017, 117, 42484286.

(3) Lee, S. S.; Loo, Y.-L. Structural Complexities in the Active Layers of Organic Electronics. Annu. Rev. Chem. Biomol. Eng. 2010, 1, 59-78.

(4) Dienel, T.; Loppacher, C.; Mannsfeld, S. C. B.; Forker, R.; Fritz, T. Growth-Mode-Induced Narrowing of Optical Spectra of an Organic Adlayer. Adv. Mater. 2008, 20, 959-963.

(5) Luo, Y.; Chen, G.; Zhang, Y.; Zhang, L.; Yu, Y.; Kong, F.; Tian, X.; Zhang, Y.; Shan, C.; Luo, Y.; Yang, J.; Sandoghdar, V.; Dong, Z.; Hou, J. G. Electrically Driven Single-Photon Superradiance from Molecular Chains in a Plasmonic Nanocavity. Phys. Rev. Lett. 2019, 122, 233901.

(6) Yamagata, H.; Spano, F. C. Strong Photophysical Similarities between Conjugated Polymers and J-Aggregates. J. Phys. Chem. Lett. 2014, 5, 622-632.

(7) Yamagata, H.; Pochas, C. M.; Spano, F. C. Designing J- and HAggregates through Wave Function Overlap Engineering: Applications to Poly(3-Hexylthiophene). J. Phys. Chem. B 2012, 116, 1449414503.

(8) Thiessen, A.; Vogelsang, J.; Adachi, T.; Steiner, F.; Vanden Bout, D.; Lupton, J. M. Unraveling the Chromophoric Disorder of Poly(3Hexylthiophene). Proc. Natl. Acad. Sci. U. S. A. 2013, 110, E3550.

(9) Lupton, J. M. Single-Molecule Spectroscopy for Plastic Electronics: Materials Analysis from the Bottom-Up. Adv. Mater. 2010, 22, 1689-1721.

(10) Adachi, T.; Lakhwani, G.; Traub, M. C.; Ono, R. J.; Bielawski, C. W.; Barbara, P. F.; Vanden Bout, D. A. Conformational Effect on 
Energy Transfer in Single Polythiophene Chains. J. Phys. Chem. B 2012, 116, 9866-9872.

(11) Clark, J.; Silva, C.; Friend, R. H.; Spano, F. C. Role of Intermolecular Coupling in the Photophysics of Disordered Organic Semiconductors: Aggregate Emission in Regioregular Polythiophene. Phys. Rev. Lett. 2007, 98, 206406.

(12) Paquin, F.; Yamagata, H.; Hestand, N. J.; Sakowicz, M.; Bérubé, N.; Côté, M.; Reynolds, L. X.; Haque, S. A.; Stingelin, N.; Spano, F. C.; Silva, C. Two-Dimensional Spatial Coherence of Excitons in Semicrystalline Polymeric Semiconductors: Effect of Molecular Weight. Phys. Rev. B: Condens. Matter Mater. Phys. 2013, 88, 155202.

(13) Yamagata, H.; Spano, F. C. Interplay between Intrachain and Interchain Interactions in Semiconducting Polymer Assemblies: The HJ-Aggregate Model. J. Chem. Phys. 2012, 136, 184901.

(14) Baghgar, M.; Labastide, J.; Bokel, F.; Dujovne, I.; McKenna, A.; Barnes, A. M.; Pentzer, E.; Emrick, T.; Hayward, R.; Barnes, M. D. Probing Inter- and Intrachain Exciton Coupling in Isolated Poly(3Hexylthiophene) Nanofibers: Effect of Solvation and Regioregularity. J. Phys. Chem. Lett. 2012, 3, 1674-1679.

(15) Kanemoto, K.; Sudo, T.; Akai, I.; Hashimoto, H.; Karasawa, T.; Aso, Y.; Otsubo, T. Intrachain Photoluminescence Properties of Conjugated Polymers as Revealed by Long Oligothiophenes and Polythiophenes Diluted in an Inactive Solid Matrix. Phys. Rev. B: Condens. Matter Mater. Phys. 2006, 73, 235203.

(16) Raithel, D.; Baderschneider, S.; de Queiroz, T. B.; Lohwasser, R.; Köhler, J.; Thelakkat, M.; Kümmel, S.; Hildner, R. Emitting Species of Poly(3-Hexylthiophene): From Single, Isolated Chains to Bulk. Macromolecules 2016, 49, 9553-9560.

(17) Raithel, D.; Simine, L.; Pickel, S.; Schötz, K.; Panzer, F.; Baderschneider, S.; Schiefer, D.; Lohwasser, R.; Köhler, J.; Thelakkat, M.; Sommer, M.; Köhler, A.; Rossky, P. J.; Hildner, R. Direct Observation of Backbone Planarization via Side-Chain Alignment in Single Bulky-Substituted Polythiophenes. Proc. Natl. Acad. Sci. U. S. A. 2018, 115, 2699.

(18) Knudsen, J. B.; Liu, L.; Bank Kodal, A. L.; Madsen, M.; Li, Q.; Song, J.; Woehrstein, J. B.; Wickham, S. F. J.; Strauss, M. T.; Schueder, F.; Vinther, J.; Krissanaprasit, A.; Gudnason, D.; Smith, A. A. A.; Ogaki, R.; Zelikin, A. N.; Besenbacher, F.; Birkedal, V.; Yin, P.; Shih, W. M.; et al. Routing of Individual Polymers in Designed Patterns. Nat. Nanotechnol. 2015, 10, 892-898.

(19) Zessin, J.; Fischer, F.; Heerwig, A.; Kick, A.; Boye, S.; Stamm, M.; Kiriy, A.; Mertig, M. Tunable Fluorescence of a Semiconducting Polythiophene Positioned on DNA Origami. Nano Lett. 2017, 17, $5163-5170$.

(20) Kasha, M. Energy Transfer Mechanisms and the Molecular Exciton Model for Molecular Aggregates. Radiat. Res. 1963, 20, 5570 .

(21) Davydov, A. S. The Theory of Molecular Excitons. Phys.Uspekhi 1964, 7, 145-178.

(22) Spano, F. C.; Silva, C. H- and J-Aggregate Behavior in Polymeric Semiconductors. Annu. Rev. Phys. Chem. 2014, 65, 477500.

(23) Forker, R.; Dienel, T.; Krause, A.; Gruenewald, M.; Meissner, M.; Kirchhuebel, T.; Gröning, O.; Fritz, T. Optical Transition Energies of Isolated Molecular Monomers and Weakly Interacting Two-Dimensional Aggregates. Phys. Rev. B: Condens. Matter Mater. Phys. 2016, 93, 165426.

(24) Kerfoot, J.; Korolkov, V. V.; Nizovtsev, A. S.; Jones, R.; Taniguchi, T.; Watanabe, K.; Lesanovsky, I.; Olmos, B.; Besley, N. A.; Besley, E.; Beton, P. H. Substrate-Induced Shifts and Screening in the Fluorescence Spectra of Supramolecular Adsorbed Organic Monolayers. J. Chem. Phys. 2018, 149, 054701.

(25) Zhao, H.; Zhao, Y.; Song, Y.; Zhou, M.; Lv, W.; Tao, L.; Feng, Y.; Song, B.; Ma, Y.; Zhang, J.; Xiao, J.; Wang, Y.; Lien, D.-H.; Amani, M.; Kim, H.; Chen, X.; Wu, Z.; Ni, Z.; Wang, P.; Shi, Y.; et al. Strong Optical Response and Light Emission from a Monolayer Molecular Crystal. Nat. Commun. 2019, 10, 5589.

(26) Alkhamisi, M.; Korolkov, V. V.; Nizovtsev, A. S.; Kerfoot, J.; Taniguchi, T.; Watanabe, K.; Besley, N. A.; Besley, E.; Beton, P. H.
The Growth and Fluorescence of Phthalocyanine Monolayers, Thin Films and Multilayers on Hexagonal Boron Nitride. Chem. Commun. 2018, 54, 12021-12024.

(27) Svatek, S. A.; Kerfoot, J.; Summerfield, A.; Nizovtsev, A. S.; Korolkov, V. V.; Taniguchi, T.; Watanabe, K.; Antolín, E.; Besley, E.; Beton, P. H. Triplet Excitation and Electroluminescence from a Supramolecular Monolayer Embedded in a Boron Nitride Tunnel Barrier. Nano Lett. 2020, 20, 278-283.

(28) Zhang, L.; Yu, Y.-J.; Chen, L.-G.; Luo, Y.; Yang, B.; Kong, F.-F.; Chen, G.; Zhang, Y.; Zhang, Q.; Luo, Y.; Yang, J.-L.; Dong, Z.-C.; Hou, J. G. Electrically Driven Single-Photon Emission from an Isolated Single Molecule. Nat. Commun. 2017, 8, 580.

(29) Zhang, Y.; Luo, Y.; Zhang, Y.; Yu, Y.-J.; Kuang, Y.-M.; Zhang, L.; Meng, Q.-S.; Luo, Y.; Yang, J.-L.; Dong, Z.-C.; Hou, J. G. Visualizing Coherent Intermolecular Dipole-Dipole Coupling in Real Space. Nature 2016, 531, 623.

(30) Kimura, K.; Miwa, K.; Imada, H.; Imai-Imada, M.; Kawahara, S.; Takeya, J.; Kawai, M.; Galperin, M.; Kim, Y. Selective Triplet Exciton Formation in a Single Molecule. Nature 2019, 570, 210-213.

(31) Doppagne, B.; Neuman, T.; Soria-Martinez, R.; López, L. E. P.; Bulou, H.; Romeo, M.; Berciaud, S.; Scheurer, F.; Aizpurua, J.; Schull, G. Single-Molecule Tautomerization Tracking through Space- and Time-Resolved Fluorescence Spectroscopy. Nat. Nanotechnol. 2020, $15,207-211$.

(32) Chen, G.; Luo, Y.; Gao, H.; Jiang, J.; Yu, Y.; Zhang, L.; Zhang, Y.; Li, X.; Zhang, Z.; Dong, Z. Spin-Triplet-Mediated Up-Conversion and Crossover Behavior in Single-Molecule Electroluminescence. Phys. Rev. Lett. 2019, 122, 177401.

(33) Korolkov, V. V.; Summerfield, A.; Murphy, A.; Amabilino, D. B.; Watanabe, K.; Taniguchi, T.; Beton, P. H. Ultra-High Resolution Imaging of Thin Films and Single Strands of Polythiophene Using Atomic Force Microscopy. Nat. Commun. 2019, 10, 1537.

(34) Eisfeld, A.; Marquardt, C.; Paulheim, A.; Sokolowski, M. Superradiance from Two Dimensional Brick-Wall Aggregates of Dye Molecules: The Role of Size and Shape for the Temperature Dependence. Phys. Rev. Lett. 2017, 119, 097402.

(35) Eder, T.; Vogelsang, J.; Bange, S.; Remmerssen, K.; Schmitz, D.; Jester, S.-S.; Keller, T. J.; Höger, S.; Lupton, J. M. Interplay between $\mathrm{J}$ - and H-Type Coupling in Aggregates of $\pi$-Conjugated Polymers: A Single-Molecule Perspective. Angew. Chem., Int. Ed. 2019, 58, 18898-18902.

(36) Niles, E. T.; Roehling, J. D.; Yamagata, H.; Wise, A. J.; Spano, F. C.; Moulé, A. J.; Grey, J. K. J-Aggregate Behavior in Poly-3Hexylthiophene Nanofibers. J. Phys. Chem. Lett. 2012, 3, 259-263.

(37) Yamagata, H.; Spano, F. C. Vibronic Coupling in Quantum Wires: Applications to Polydiacetylene. J. Chem. Phys. 2011, 135, 054906.

(38) Athanasopoulos, S.; Hoffmann, S. T.; Bässler, H.; Köhler, A.; Beljonne, D. To Hop or Not to Hop? Understanding the Temperature Dependence of Spectral Diffusion in Organic Semiconductors. J. Phys. Chem. Lett. 2013, 4, 1694-1700.

(39) Guha, S.; Rice, J. D.; Yau, Y. T.; Martin, C. M.; Chandrasekhar, M.; Chandrasekhar, H. R.; Guentner, R.; Scanduicci de Freitas, P.; Scherf, U. Temperature-Dependent Photoluminescence of Organic Semiconductors with Varying Backbone Conformation. Phys. Rev. B: Condens. Matter Mater. Phys. 2003, 67, 125204.

(40) Louarn, G.; Buisson, J. P.; Lefrant, S.; Fichou, D. Vibrational Studies of a Series of $\alpha$-Oligothiophenes as Model Systems of Polythiophene. J. Phys. Chem. 1995, 99, 11399-11404.

(41) Spano, F. C. The Spectral Signatures of Frenkel Polarons in Hand J-Aggregates. Acc. Chem. Res. 2010, 43, 429-439.

(42) Parkinson, P.; Müller, C.; Stingelin, N.; Johnston, M. B.; Herz, L. M. Role of Ultrafast Torsional Relaxation in the Emission from Polythiophene Aggregates. J. Phys. Chem. Lett. 2010, 1, 2788-2792.

(43) Deckers, S.; Vandendriessche, S.; Cornelis, D.; Monnaie, F.; Koeckelberghs, G.; Asselberghs, I.; Verbiest, T.; van der Veen, M. A. Poly(3-Alkylthiophene)s Show Unexpected Second-Order Nonlinear Optical Response. Chem. Commun. 2014, 50, 2741-2743. 
(44) Britnell, L.; Gorbachev, R. V.; Jalil, R.; Belle, B. D.; Schedin, F.; Mishchenko, A.; Georgiou, T.; Katsnelson, M. I.; Eaves, L.; Morozov, S. V.; Peres, N. M. R.; Leist, J.; Geim, A. K.; Novoselov, K. S.; Ponomarenko, L. A. Field-Effect Tunneling Transistor Based on Vertical Graphene Heterostructures. Science 2012, 335, 947.

(45) Withers, F.; Del Pozo-Zamudio, O.; Mishchenko, A.; Rooney, A. P.; Gholinia, A.; Watanabe, K.; Taniguchi, T.; Haigh, S. J.; Geim, A. K.; Tartakovskii, A. I.; Novoselov, K. S. Light-Emitting Diodes by Band-Structure Engineering in van der Waals Heterostructures. Nat. Mater. 2015, 14, 301-306.

(46) Binder, J.; Howarth, J.; Withers, F.; Molas, M. R.; Taniguchi, T.; Watanabe, K.; Faugeras, C.; Wysmolek, A.; Danovich, M.; Fal'ko, V. I.; Geim, A. K.; Novoselov, K. S.; Potemski, M.; Kozikov, A. Upconverted Electroluminescence via Auger Scattering of Interlayer Excitons in van der Waals Heterostructures. Nat. Commun. 2019, 10, 2335.

(47) Khestanova, E.; Guinea, F.; Fumagalli, L.; Geim, A. K.; Grigorieva, I. V. Universal Shape and Pressure inside Bubbles Appearing in van der Waals Heterostructures. Nat. Commun. 2016, 7, 12587.

(48) Vasu, K. S.; Prestat, E.; Abraham, J.; Dix, J.; Kashtiban, R. J.; Beheshtian, J.; Sloan, J.; Carbone, P.; Neek-Amal, M.; Haigh, S. J.; Geim, A. K.; Nair, R. R. Van der Waals Pressure and Its Effect on Trapped Interlayer Molecules. Nat. Commun. 2016, 7, 12168.

(49) de Melo, J. S.; Silva, L. s. M.; Arnaut, L. s. G.; Becker, R. S. Singlet and Triplet Energies of $\alpha$-Oligothiophenes: A Spectroscopic, Theoretical, and Photoacoustic Study: Extrapolation to Polythiophene. J. Chem. Phys. 1999, 111, 5427-5433.

(50) Rossel, F.; Pivetta, M.; Schneider, W.-D. Luminescence Experiments on Supported Molecules with the Scanning Tunneling Microscope. Surf. Sci. Rep. 2010, 65, 129-144.

(51) Larkin, J. M.; Donaldson, W. R.; Foster, T. H.; Knox, R. S. Reverse Intersystem Crossing from a Triplet State of Rose Bengal Populated by Sequential 532- + 1064-nm Laser Excitation. Chem. Phys. 1999, 244, 319-330.

(52) Partee, J.; Frankevich, E. L.; Uhlhorn, B.; Shinar, J.; Ding, Y.; Barton, T. J. Delayed Fluorescence and Triplet-Triplet Annihilation in Pi-Conjugated Polymers. Phys. Rev. Lett. 1999, 82, 3673-3676.

(53) Thomas, A. K.; Garcia, J. A.; Ulibarri-Sanchez, J.; Gao, J.; Grey, J. K. High Intrachain Order Promotes Triplet Formation from Recombination of Long-Lived Polarons in Poly(3-Hexylthiophene) JAggregate Nanofibers. ACS Nano 2014, 8, 10559-10568.

(54) Pizzocchero, F.; Gammelgaard, L.; Jessen, B. S.; Caridad, J. M.; Wang, L.; Hone, J.; Bøggild, P.; Booth, T. J. The Hot Pick-Up Technique for Batch Assembly of van der Waals Heterostructures. Nat. Commun. 2016, 7, 11894.

(55) Castellanos-Gomez, A.; Buscema, M.; Molenaar, R.; Singh, V.; Janssen, L.; van der Zant, H. S. J.; Steele, G. A. Deterministic Transfer of Two-Dimensional Materials by All-Dry Viscoelastic Stamping. 2D Mater. 2014, 1, 011002. 